\section{RESILIENCIA COMUNITARIA Y SENTIDO DE COMUNIDAD DURANTE LA RESPUESTA Y RECUPERACIÓN AL TERREMOTO- TSUNAMI DEL AÑO 2010, TALCAHUANO-CHILE}

Mauricio Torres Méndez ${ }^{*}$, Beatriz Cid Aguayo ${ }^{2}$, María Teresa Bull ${ }^{3}$, Jenny Moreno ${ }^{4}$, Alejandro Lara ${ }^{5}$, Carlos Gonzalez Aburto ${ }^{1}$ y Bárbara Henríquez Arriagada ${ }^{6}$

\section{RESUMEN}

La investigación, tiene entre sus objetivos, describir cómo se expresan las capacidades de afrontamiento de la resiliencia comunitaria en relación al sentido de comunidad en trece familias de la comuna de Talcahuano - Chile, durante el periodo de respuesta y recuperación al desastre del terremoto y tsunami del año 2010. Se optó por un diseño exploratorio cualitativo de carácter transversal bajo una lógica de observación ex-post. A los casos de estudio se les aplica una entrevista semi-estructurada, cuyos discursos son examinados utilizando el Análisis Estructural de Contenido y el Modelo Actancial de la Semántica Estructural. Se concluye que la resiliencia comunitaria es un proceso que emerge en vinculación con el sentido de comunidad, expresado en el compromiso por el bienestar de la comunidad del lugar y en la participación comunitaria en acciones colectivas. Estas son condicionantes sociales que facilitan o limitan la adaptación organizativa de la comunidad y el surgimiento de comportamientos colaborativos, en función de resolver los problemas con mayor rapidez y con el mejor desempeño en el uso de los recursos del territorio.

\section{PALABRAS CLAVE}

Resiliencia comunitaria, Vulnerabilidad social ante desastres, Capacidades de afrontamiento, Sentido de comunidad, Reducción del Riesgo de Desastre

\section{COMMUNITY RESILIENCE AND SENSE OF COMMUNITY DURING THE RESPONSE AND RECOVERY TO THE 2010 EARTHQUAKE-TSUNAMI, TALCAHUANO-CHILE}

\section{ABSTRACT}

The research aims to analyze how the coping capacities and community resilience are expressed in relation to the sense of community in thirteen families of the commune of Talcahuano in Chile, specifically during the period of response and recovery to the earthquake and tsunami disaster occurred in 2010. We opted for a transversal and qualitative exploratory design under the logic of ex-post observation. Semi-structured interviews were conducted and subsequent discourses examined using Structural Content Analysis and the Actantial Model of Structural Semantics. We conclude that community resilience in Talcahuano emerges in connection with the sense of community, expressed through the community's commitment for well-being and into collective actions. These social arrangements can facilitate or limit the organizational adaptability of the community as well as the development of collaborative behaviors. Such adaptability and behavior are relevant to solve problems quicker and with a more efficient use of the territory's resources.

\section{KEYWORDS}

Community resilience, Social vulnerability to disasters, Coping capacities, Sense of community, Disaster Risk Reduction
1. Magíster en Investigación Social y Desarrollo, Universidad de Concepción, Chile.

2. Departamento de Sociología y Antropología, Universidad de Concepción, Chile.

3. Observatorio de Gestión en Desastres, Facultad de Ingeniería, Universidad Católica de la Santisima Concepción, Chile.

4. Alliance Manchester Business School, University of Manchester, Reino Unido.

5. Departamento de Arquitectura, Universidad de Concepción, Chile.

\section{Universidad de} Concepción, Chile.

*Autor de correspondencia: matorres@udec.cl

\section{RECIBIDO}

21 de agosto de 2017

\section{ACEPTADO}

26 de septiembre de 2017

\section{PUBLICADO}

30 de enero de 2018

\section{Formato cita}

Recomendada (APA):

Torres Méndez, M., Cid Aguayo, B., Bull, M.T.,

Moreno, J., Lara, A., Gonzalez, C. y Henriquez, B. (2018). Resiliencia comunitaria y sentido de comunidad durante la respuesta y recuperación al terremoto-tsunami del año 2010, Talcahuano-Chile. REDER, 2(1), pp.21-37.

community as well as the development of collaborative behaviors. Such adaptability and behavior
are relevant to solve problems quicker and with a more efficient use of the territory's resources.
Community resilience, Social vulnerability to disasters, Coping capacities, Sense of community,
Disaster Risk Reduction

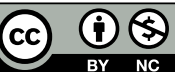

Todos los artículos publicados en REDER siguen una política de Acceso Abierto y se respaldan en una Licencia CreativeCommons Atribución-NoComercial 4.0 Internacional.

Revista de Estudios Latinoamericanos sobre Reducción del Riesgo de Desastres (REDER) 


\section{INTRODUCCIÓN}

El contexto de la discusión sobre la resiliencia comunitaria ha transitado desde un enfoque centrado en la naturalidad de las amenazas y las capacidades reactivas de institucionales de respuesta (Cannon, 1994), hacia uno donde la mirada se centra en las condiciones de vulnerabilidad (Wisner et al., 2004; Cutter et al., 2003, 2008) que obstaculizan el desarrollo de capacidades comunitarias (Smit y Wandel, 2006) vinculadas a la resiliencia social ante desastre.

Esta investigación vincula el sentido de comunidad con el desempeño de las capacidades de afrontamiento de la resiliencia comunitaria ante desastres, al facilitar comportamientos colaborativos con estilos de resolución de problemas caracterizados por la delegación de responsabilidades y el uso de los recursos del territorio.

La investigación busca ampliar los conocimientos sobre las capacidades comunitarias que ayudan a afrontar los desastres, promoviendo el desarrollo de medidas preventivas y cambios estructurales (Wilson, 2012) que integren aspectos vinculados a la participación comunitaria, el compromiso por el bienestar de los miembros de la comunidad, el sentido de pertenencia y el manejo de los estilos de resolución de problemas. De manera tal, los habitantes se preparen cooperativa y anticipadamente para operar de una manera más autónoma durante un desastre (Drabek, 2014 en Rodríguez, 2014, p. 220)

No obstante, la escasa preparación institucional y comunitaria para afrontar la crisis vinculada a la seguridad, el abastecimiento de alimento, agua e información fue una característica del desastre iniciado por el terremoto y tsunami el día 27 de febrero del año 2010. Esta situación toma aún más importancia, al considerar que desde esa fecha, en el país se han experimentado al menos trece desastres de amplia cobertura, los que dejan una frecuencia promedio aproximada de un desastre cada seis a siete meses'.

Respondiendo a las características de la pregunta de investigación y al objeto de estudio, se utiliza un diseño descriptivo de carácter transversal, bajo una lógica de observación ex-post. En vista a lo cual se aplican entrevistas semiestructuradas a los trece casos estudiados del territorio de Las Salinas en la comuna de Talcahuano, con el objeto de reconstruir los diferentes momentos y los sistemas de sentido de los relatos, desde el mismo momento del terremoto hasta el término del periodo de recuperación.

El análisis toma herramientas de la semántica estructural (Greimas, 1971) para determinar los campos de acción y del análisis estructural de contenido (Hiernaux, 1996) para definir el contenido y las estructuras de las significaciones de los relatos de los casos de estudio. Esta permite el análisis de una manera objetiva y sistemática, observando los procesos mediante la clasificación de las codificaciones (Vieytes, 2004) de acuerdo al periodo post-desastre y los componentes de la resiliencia comunitaria.

Los resultados de la investigación dan cuenta del distanciamiento entre los avances en los acuerdos de las organizaciones internacionales en la materia (UNISDR, 2005, p. 7; UNISDR, 2015), y el fortalecimiento de las capacidades comunitarias para afrontar y adaptarse a nuevas crisis (Cannon, 1994; Dombrowsky, 1995). Por lo que se requiere de la integración de la población en los procesos de gestión de riesgo para reducir el uso de formas inadecuadas para medir el riesgo y la efectividad de las decisiones políticas (Cardona, 2004 en Bankoff, 2004, P.51).

\section{Territorio Las Salinas, Comuna Talcahuano}

El área seleccionada para la aplicación de la investigación es el territorio de Las Salinas (Figura 1), que pertenece a la comuna de Talcahuano. En la ciudad hubo 53.637 personas damnificadas, 33 fallecidos, 1.956 viviendas destruidas y 11.514 viviendas con daño diverso; además diversos daños en edificios públicos, infraestructura urbana, industrial y productiva artesanal como son las embarcaciones. Luego del terremoto y tsunami, se produjo el corte en los suministros de agua, electricidad, gas y telefonía, desabastecimiento de víveres y combustibles. Hubo daño en las plantas de tratamiento y colectores de aguas servidas, las que junto a la interrupción del sistema de

1. Los eventos considerados son: La sequía más severa registrada en la zona central desde el año 2010 (Cisternas, 2016), la erupción del complejo volcánico Puyehue-Cordón Caulle el año 2011, el terremoto de Zapallar el año 2012, el terremoto de Vallenar el año 2013, el terremoto y tsunami de Iquique el año 2014, el incendio de los Cerros de Valparaíso el año 2014, el temporal y aluvión del Norte de Chile el año 2015, la Erupción del Volcán Calbuco el año 2015, el terremoto y tsunami de Coquimbo el año 2015, la contaminación de la Marea Roja el año 2016 en Chiloé, los incendios forestales de enero del 2017 que consumieron aproximadamente 500.ooo hectáreas principalmente de monocultivo forestal, el aluvión de Los Andes a fines de febrero del 2017 que afecto la región metropolitana y el desbord del rio Salado producto del evento hidrometeorológico que afectó a la región de Atacama durante el mes de mayo del 2017. 
recolección de basura y la deposición del material orgánico arrastrado por el tsunami, se provocó una condición de insalubridad en los sectores afectados. El tsunami destruyó las embarcaciones de la bahía de San Vicente y Concepción, del sector Las Salinas y gran parte del sector Centro, en las caletas El Soldado y Tumbes, quedando inhabilitadas otras tres caletas de pescadores.

El territorio de Las Salinas resulta ser afectado en su totalidad por el desastre del año 2010 (Figura 1), siendo impactada por un tren de olas durante varias horas después del terremoto. Su población evacuó, para luego retornar a sus viviendas destruidas. La principal actividad del territorio es residencial, albergando a 27.718 habitantes, que representan al $17 \%$ de la población comunal total (INE, 2002). De los cuales un $52 \%$ son mujeres y un $48 \%$, hombres. El sector cuenta con al menos 3.206 adultos mayores de 60 años que corresponden aproximadamente al $12 \%$ de los habitantes de todo el sector. Está ordenado en 9 Unidades Vecinales, que congregan 32 Juntas de Vecinos y 223 organizaciones funcionales entre Comités de Adelanto y/o Pavimentación, Organizaciones Deportivas y Centros culturales y artísticos.

En cuanto a los recursos físicos y estructuras críticas, el territorio cuenta con un Centro de Salud Familiar con 15.621 inscritos válidos, equivalente a la mitad de la población estimada del sector. Además se emplaza en la localidad el cuartel de la Cuarta Compañía de Bomberos de Talcahuano, dos servicentros, dos hogares de ancianos; los Gimnasios Los Cóndores y Rey Barón; las Iglesias Santa Cecilia y Volga; el Club Deportivo el Manzano; dos supermercados; un taller municipal donde se guardan las maquinarias de la Dirección de Aseo y Ornato; dos liceos públicos de educación superior, entre otros.

\section{Amenaza de terremoto y tsunami}

El terremoto y tsunami que afectó a la comuna de Talcahuano madrugada del 27 de febrero del año 2010, tuvo una magnitud de 8.8 grados en la escala de Richter, ocasionado por un proceso de subducción interplaca de 66 milímetros por año (Angerman, 1999), implicó una ruptura longitudinal de $500 \mathrm{~km}$. y $150 \mathrm{~km}$. de ancho, la que se propagó bilateralmente al norte y sur del epicentro (Melnick, 2012). Predecesor directo de este evento, por las características y ubicación, fue el terremoto y tsunami de 1835, evento registrado por Robert FitzRoy y Charles Darwin, quienes documentaron un aumento entre 2.4 a 3 metros en la altura de las costas de la Isla Santa María. Durante los siguientes 175 años, la isla experimentó un hundimiento alrededor de 1,4 metros y para el 2010 un nuevo aumento de la altura de costa de 1,8 metros. Constatándose un patrón de deformación vertical en las costas (Wesson et al. 2015). De igual forma Talcahuano experimentó un desplazamiento aproximado de 3 metros hacia el Este, registrándose un peak de desplazamiento de 5 metros al norte de la Comuna de Constitución y la Isla Santa María (Moreno et al, 2012).

En Chile se pueden presentar dos tipos de recurrencia sísmica, entre 80 a 100 años para los mayores de magnitud 7.5 (Cisternas et al., 2012, p.24) y entre 250 a 500 años para los superiores a 8.5 (Cisternas et al., 2005), siendo un desafío mantener la memoria preventiva para este tipo de ocurrencia, como se observó el año 2010.

\section{MARCO REFERENCIAL}

Los planteamientos teóricos que se expresan a continuación, inician con la discusión sobre la comprensión de las vulnerabilidades sociales que determinan los desastres y la gestión del riesgo de desastre desde la acción política y pública. De lo anterior, se desprende la discusión teórica respecto a los atributos que definen la resiliencia social al interior de un grupo de personas u organizaciones, focalizando la mirada sobre las capacidades comunitarias para responder y ajustarse a los cambios generados por los desastres. Finalmente se plantea que la resiliencia social requiere de ciertas condiciones comunitarias vinculadas con el sentido de comunidad para lograr un mayor desempeño en el uso de los recursos locales durante el desastre.

\section{Reducir la vulnerabilidad para gestionar el riesgo}

La comprensión del desastre al interior de la discusión académica comienza a cambiar luego de la segunda guerra mundial, producto de visiones críticas al sesgo naturalista que le otorgaba a las variables físicas gran parte de su explicación (Drabek 1970; Cannon, 1994; Wisner, 2004; 
Fordham, 2014; en Bankoff et al., 2014). En este proceso se pueden distinguir dos paradigmas de interrelación conceptual, el paradigma dominante (Tierney, 2002; en Stallings, 2002; Tierney, 2014), también denominado "comand and control" (Fordham 2004 en Bankoff et al., 2004 p. 175), cuyo principal objetivo es retornar a la normalidad luego de un desastre, priorizando la respuesta centralizada y jerarquizada; y el paradigma de la vulnerabilidad social (Mileti, 1999; Thomas et al. 2013), cuyo enfoque conduce hacia la participación activa de los diferentes grupos sociales en el aseguramiento del derecho de todos a sobrevivir y la protección de la calidad del medioambiente con el objeto de reducir los impactos sobre la población con menos recursos.

En esta última instancia, el grado de afectación que una amenaza logra producir en un grupo o sociedad, se relaciona con los componentes humanos que construyen la vulnerabilidad social frente una situación de estrés, siendo la pobreza la causa raíz más importante a largo plazo (Zakour, 2013). No obstante, la vulnerabilidad no se limita a la temática de la pobreza, ya que diferentes aspectos influyen en que un grupo de personas sea más afectado ante una crisis.

Operacionalmente, existe una variedad de mediciones, representaciones y cuantificaciones de la vulnerabilidad social ante desastre (Cutter, 2003; Laska et al., 2005; Emrich et al., 2011; Holland et al., 2011 en Singh, 2014; Zakour et al., 2013; Bergstrand et al., 2014; Lee et al., 2014; Singh, 2014; Fakhruddin et al., 2015) en las que se observa un consenso respecto a la importancia de los principales factores: edad, ingreso, salud y nivel educacional.

Se logra un consenso respecto a que los desastres se gestan desde la construcción continua de las brechas del desarrollo y el aumento de la exposición económica y demográfica (EIRD/ONU, 2011). De tal manera, los riesgos y los desastre son entendidos como construcciones sociales que deben trabajarse desde su producción y no sólo desde sus efectos, mitigando los efectos adversos antes de que ocurran los desastres (Mileti, 1999). Sin embargo, la mayoría de las investigaciones no integra el análisis de los procesos sociales que generan la vulnerabilidad en las localidades, focalizando sus objetivos en la presencia, peso y distribución de ciertos factores.

El proceso anterior alimenta visiones críticas respecto a la efectividad de las acciones orientadas para la reducción del riesgo de desastre. Por lo que se proponen gestiones transversales e interdisciplinarias que involucren a las comunidades en tres tipos de políticas públicas: la identificación del riesgo, la reducción del riesgo y la gestión del desastre (Cardona 2004 en Bankoff et al. 2004, p. 40). Esta diferenciación se asemeja a dos grandes procesos continuos (Narváez et al., 2009 , p. 33) de los que depende la gestión del riesgo de desastre, aquellos relacionados a la reducción del riesgo y los vinculados a la gestión del desastre.

\section{Los atributos de la resiliencia comunitaria}

El concepto de la resiliencia comunitaria, que se desprende de los planteamiento sobre resiliencia socioecológica (Holling, 1973; Werner and Smith, 1982; Werner and Smith, 1992, en Quesada, 2002; Folke, 2006; Norris et al., 2007; Wamsler, 2014), se enfoca en la habilidad organizativa (Tobin, 1999 en Ainuddin y Routray, 2012, p. 26) de los integrantes de la comunidad para hacer uso eficiente (Paton et al., 2001, p. 159; Patterson et al., 2009; Cohen et al., 2016) de las capacidades de afrontamiento (Turner et al., 2003; Twigg, 2007; Boin y Hart 2014, en Rodriguez et al., 2014; Engel, 2015; Parsons et al., 2016) y las capacidades adaptativas (Smit y Wandel, 2006; Longstaff, 2010; Keck y Sakdapolak, 2013) para reducir las vulnerabilidades ante desastres (Adger et al., 2004, 2006; Cutter et al., 2008, 2009).

En este enfoque, las comunidades son el lugar de respuesta para cada unidad social (Dynes, 2005; Twigg, 2007) durante diferentes crisis. A través de la agencia comunitaria (Manyena et al., 2011, p. 419), en base a sus propios intereses, preferencias y recursos comunitarios (Patterson et al., 2009; Pfefferbaum et al., 2015: p. 239), los integrantes de una comunidad logran cambiar las condiciones que generan su vulnerabilidad, promoviendo acciones para incrementar su resiliencia (Berkes and Roos, 2013; Djalante et al. 2011, p. 350) a través de esfuerzos colectivos y estratégicos para invertir los recursos comunitarios en el desarrollo de capacidades de afrontamiento y adaptación (Flora and Flora, 2004, citado por Magis, 2010). 
Por lo que, las personas de una comunidad son resilientes juntos y no simplemente de una manera similar (Norris et al., 2007). En efecto, los recursos personales y privados utilizados durante un desastre pueden no contribuir a la resiliencia comunitaria. El acoplamiento estratégico de los recursos desde la acción colectiva, logra un mejor desempeño en las acciones para reducir los daños (Magis, 2010, pp. 410-411). Una comunidad resiliente requiere de un trabajo conjunto a través de comportamientos altruistas y de relaciones sociales compartidas (Sapirstein, 2006) que permitan la continuidad del funcionamiento mediante la adaptación y la recuperación efectiva (Fraser et al., 1999).

El grado de resiliencia comunitaria se vincula con el desempeño de las capacidades para responder y elaborar estrategias eficientes para la recuperación ante un desastre, de acuerdo a cuatro propiedades de un sistema resiliente: robustez, redundancia, sinergia/recursividad y rapidez (Bruneau et al., 2003, p. 737). Estas propiedades son consideradas como atributos dinámicos de los procesos de vinculación de las capacidades adaptativas que influyen en la eficacia de la respuesta de una población frente a situaciones de estrés o desastre (Norris et al., 2008). Por consiguiente, la robustez se relaciona a la habilidad para resistir, y soportar un estrés sin degradación o pérdida de funciones, mientras que la redundancia se vincula con el grado en que un elemento es sustituible por otro con el objeto tener más de una vía para resolver un problema, entendiéndola como el opuesto de la dependencia (Norris et al. 2008, p. 134)

La resiliencia comunitaria no es solo teórica, sino que debe estar vinculada de igual forma con la acción práctica y colectiva (Magis, 2010) para lograr un mayor desempeño de las capacidades adaptativas (Norris, 2007). Por lo que, las acciones referidas al control del poder son tanto más relevantes que la propia capacidad de auto-organización comunitaria en situaciones de desastre. De modo que los procesos de formación de identidad y conexión comunitaria, la vinculación con el lugar, los liderazgos y la participación (Berkes and Ross et al., 2013, p. 12) logran importancia en el análisis de las capacidades de afrontamiento y adaptación vinculadas con la resiliencia comunitaria.

\section{Sentido de comunidad, un condicionante social para una comunidad resiliente}

Las capacidades comunitarias para afrontar y adaptarse a situaciones de estrés, están influidas por características personales, componentes cognitivos y prácticas comunitarias durante la respuesta y la recuperación (Tobin, 1999; citado en Paton et al., 2000). Por lo que, la resiliencia comunitaria ante desastre se vincula estrechamente con las condiciones sociales que permiten el mayor desempeño en la toma de decisiones para afrontar los problemas durante el desastre, la mitigación de las consecuencias adversas y en las acciones para potenciar la adaptación durante la recuperación.

En este aspecto, el sentido de comunidad es una de las condiciones que facilita el desarrollo de la resiliencia comunitaria ante desastre y se define por el sentimiento de adhesión a personas y a lugares, lo que estimula la participación en respuestas comunitarias al desastre y al acceso a la red de soporte social (Paton et al., 2001, p. 159), siendo incluso determinante de la cohesión social (Chang, 2010, p.298). Además, tiene estrecha relación con la eficiencia y la eficacia de los procesos de recuperación (Aldrich, 2012), mediante el soporte de las capacidades comunitarias en la coordinación y cooperación para la transferencia de información e ideas sobre la resolución de problemas, la entrega de ayuda financiera, la facilitación de herramientas y la habilitación de un lugar para vivir, entre otros

En la investigación, el sentido de comunidad (McMillan y Chaves, 1989, p.9; Paton et al. 2001, p.159; Smit y Wandel, 2006, p. 285; Norris, 2007, p. 139; Kulig et al. 2008 citado en Ainuddin et al. 2012. p.28; Wamsler, 2014; Kenney, 2015, p.48) está definido por los vínculos sociales, el sentimiento de pertenencia (Wilson, 2012), el compromiso por los problemas comunitarios, y la participación comunitaria en acciones colectivas centrados en los problemas (Bachrach y Zautra, 1985; citado McMillan y Chaves, 1989, p.7) durante la respuesta a un desastre. Esto involucra la conformación de lazos de confianza, incluyendo valores compartidos (Perkins et al., 2002). El sentido de comunidad puede transcender el nivel relacional-afectivo entre los miembros para generar una vinculación mayor con el ambiente físico (Altman and Low, 2012). Este nexo estimula la participación de acciones colectivas para la respuesta comunitaria durante un desastre, tal como lo demostró Chamlee wright y Storr (2009) en su investigación post Huracán Katrina. 
Adicionalmente, entre las condicionales sociales de la resiliencia comunitaria ante desastre se menciona el estilo de afrontamiento y la autoeficacia, el primero, relacionado con la resolución de los problemas mediante el uso de los recursos locales y comunitarios (Dynes, 1994; Tierney 2003, p. 40), mientras que la auto-eficacia es definida como la capacidad de desempeño, receptividad de la información y probabilidad de adoptar comportamientos favorables para la reducción del riesgo (Paton et al., 2001).

Ainuddin y Routray (2012) agregan la percepción de riesgo como un factor clave en la definición de la resiliencia, debido al vínculo directo con el conocimiento de las amenazas y con las estrategias para la preparación. Por lo que, aquellos procesos de intervención que logran una adaptación y recuperación exitosa de las comunidades luego de una adversidad, tienden a ayudar a la población en el análisis de su ambiente, la determinación de objetivos y el desarrollo de acciones; en torno a temas comunes como el empoderamiento, la participación comunitaria y la autodeterminación (Pferfferbaum et al., 2015, p. 241).

Cabe mencionar que la traducción de las características de la resiliencia en los sistemas ecológicos (Norris, 2007) debe tener cuidado de volver a naturalizar el riesgo mediante enfoques simplistas y mecanicistas del orden social, obviando las relaciones de poder y despolitizando los mecanismos que construyen la vulnerabilidad (Keck and Sakapolrak, 2013).

\section{Periodo de respuesta y recuperación}

Una de las características de los estudios de desastre, es que el objeto de estudio (Henríquez y Barriga, 2005) transita por diferentes circunstancias y contextos, lo que exige diferenciar la fase del proceso de desastre que se investiga (Stallings, 2002). Con el objeto de ayudar a ordenar las actividades que componen los proceso continuos del desastre, desde antes del impacto de una amenaza como luego de este, se hace uso de cuatro etapas que integran el ciclo de vida del desastre (Mileti, 1999; Thomas et al., 2013; McEntire, 2015): mitigación, preparación, respuesta y recuperación. No obstante, McEntire $(2015$, p. 5) insiste en reconocer el carácter dinámico del riesgo, expresando abiertamente que las operaciones de respuesta y recuperación están presentes al mismo tiempo, por lo que es difícil determinar cuando la respuesta termina y comienza la recuperación. Por tanto, las comunidades pueden generar adaptación y fortalecer su resiliencia durante el mismo proceso de respuesta, al fomentar los vínculos y los recursos comunitarios (Magis, 2010, p. 405).

En relación a la presente investigación, el contexto del análisis se produce luego del desastre, durante los periodos de respuesta y recuperación, debido al interés por identificar las capacidades de afrontamiento y las capacidades adaptativas de la comunidad que quedan en evidencia durante la crisis que genera el desastre. El periodo de respuesta se define como aquel que sucede inmediatamente después del impacto (Mileti, 1999). En este momento las actividades consisten en salvaguardar la vida y la propiedad de las personas. El periodo puede ser tanto de corto como largo plazo (Rivera, 2015), dependiendo de las capacidades de afrontamiento de un territorio. Mientras que el periodo de recuperación ante un desastre es el momento en que las acciones se enfocan a retornar a la normalidad los estándares de los sistemas que soportan la vida, destinando esfuerzos para la reparación de las viviendas y la reconstrucción de la infraestructura dañada (McEntire, 2015). Es en este momento cuando se generan las capacidades adaptativas (Wamsler, 2015) frente a nuevos escenarios de riesgo de desastres.

\section{MÉTODO}

La investigación se ordena según un diseño descriptivo de carácter transversal, bajo una lógica de observación ex-post, utilizando como fuente primaria de información de los casos de estudio correspondientes a trece familias residentes del territorio de Las Salinas, en la comuna de Talcahuano, afectadas por el terremoto y tsunami del año 2010. A través de la aplicación de entrevistas semi-estructuradas a cada cónyuge y miembro de la familia, se extraen los relatos biográficos correspondientes a veinticinco personas respecto al periodo de respuesta y recuperación. 
El proceso de selección de las familias residentes del territorio de Las Salinas inicia con el levantamiento de información al interior del Municipio de Talcahuano, solicitando información respecto a las directivas de las Juntas de Vecinos del área de estudio y orientación respecto a las delimitaciones de cada Junta de Vecinos al interior del territorio de Las Salinas. Desde el municipio se obtiene una lista con todas las Juntas de Vecinos de la comuna y se aclara que no existe la delimitación solicitada, debido a que los cuadrantes se estructuran en base a Unidades Vecinales. Éstas pueden albergar diferentes Juntas de Vecinos, incluso solapándose entre ellas dentro de una misma Unidad Vecinal. De modo que en una misma población pueden haber varias Juntas de Vecinos, mientras que en otras ninguna. A los miembros de cada directiva se les solicita apoyo en la identificación de familias que cumplieran los criterios dispuestos por el diseño metodológico. Se agendaron entrevistas correspondientes a siete de las nueve Unidades Vecinales, producto de diferentes contratiempos con la directiva de las juntas de vecinos.

\section{Unidad de análisis}

Los discursos de familias residentes y dirigentes vecinales de las juntas de vecinos del Territorio Las Salinas, afectadas por el terremoto e inundación del tsunami del año 2010 durante el periodo de respuesta y recuperación.

\section{Unidades de Observación y criterios de selección}

La investigación cuenta con dos unidades de observación, al menos un cónyuge de las familias seleccionadas del territorio de Las Salinas, y al menos un dirigente vecinal de las juntas de vecinos correspondientes a la ubicación de las familias entrevistadas en el territorio de Las Salinas.

Los criterios de selección de las familias son que al menos un cónyuge haya estado en la vivienda durante el terremoto. En caso de una familia monoparental, que la cabeza de familia haya estado en la vivienda durante el terremoto; que las viviendas donde alojaban, hayan sido inundadas por el tsunami; y que hayan vivido sin interrupciones en sus viviendas al menos durante cinco años antes del terremoto y tsunami.

Respecto a los criterios para la selección de los dirigentes vecinales, se definió que hayan estado en ejercicio durante el periodo de respuesta y recuperación; y que el área de representación de la junta de vecinos corresponda a la de alguna de las familias seleccionadas.

La Figura 1 presenta un mapa donde se expone la distribución geográfica de los casos considerados en este estudio.

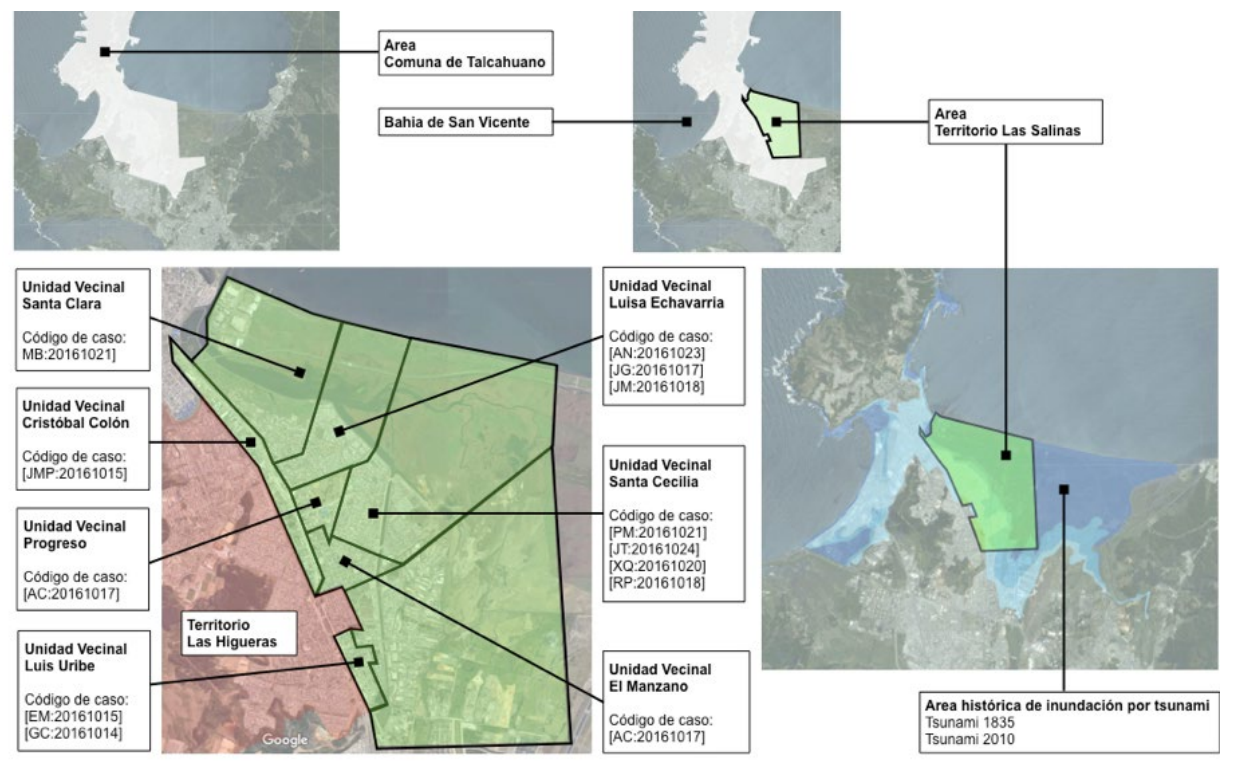

Figura 1: La distribución de los casos de estudios en el Territorio de Las Salinas sobre la comuna de Talcahuano Fuente: Autores, 2018, baseda en el Sistema de Información Geográfica para la Gestión de Riesgo del Municipio de Talcahuano. 


\section{Técnicas de análisis de información}

La información recopilada se procesó mediante un análisis de contenido, de acuerdo a la metodología propuesta por Krippendorf (2004). Este tipo de análisis comienza con la búsqueda y selección de unidades básicas de información (unitizing), en este caso, expresiones orales de los entrevistados que den indicios de capacidades de afrontamiento presentes en su experiencia con el terremoto. Luego se generan categorías a partir de estas unidades. En este caso, se crearon dos categorías: capacidades de afrontamiento durante el período de respuesta, y capacidad de afrontamiento durante el período de recuperación.

\section{EL SENTIDO DE COMUNIDAD ANTE DESASTRE}

\section{Capacidades de afrontamiento durante el periodo de respuesta}

El sentido de comunidad se vincula con el desempeño de las capacidades de afrontamiento a través del surgimiento de comportamientos colaborativos caracterizados por la delegación de responsabilidades y el uso participativo de los recursos del territorio, durante la respuesta al terremoto y tsunami y posteriormente durante la crisis que inicia luego de la evacuación.

Es así como, el sentido de pertenencia al territorio, expresado en los conocimientos y entrenamientos relacionados con las vías de evacuación y el grado de exposición a un tsunami, facilita que se generen acciones rápidas y seguras (Gaillard y Mercer, 2013) durante el desastre. Sin embargo, estos conocimientos no son generalizados al interior de la familia, de modo que por sí mismos no logran garantizar conductas seguras sin una disposición previa de la manera en la que se toman las decisiones al interior del hogar.

Así mismo, el origen de quien reside en una zona expuesta determina el grado de conocimientos sobre los saberes del territorio, los que responden a lecciones aprendidas de las experiencias en anteriores desastres (Soulé, 2014). Por lo que, quienes proceden de regiones con otras características de riesgo, presentan una mayor desventaja respecto al manejo de los criterios para una rápida identificación del riesgo, incluso aunque tengan conocimiento de las características de una amenaza.

"[...] Comenzó un movimiento, movimiento que fue en aumento y en diferentes direcciones. No sabía realmente qué pasaba. Para mí era un temblor, porque si bien nosotros hablamos de temblor y hablamos de terremoto, como aquíel terremoto es muy fuerte. Yono dimensionénunca que eso era un terremoto y que había que evacuar [...] Habían unos cubanos de arrendatarios cerca de mi casa. Éramos pocos los vecinos que estábamos en el sector" [código de caso: JG:20161017]

Esta desventaja se agrava cuando el personal de las instituciones de primera respuesta y desde una Radioemisora nacional, informan un falso estado de la amenaza, invitando a las personas evacuadas a regresar a sus viviendas expuestas al tsunami.

Estos conocimientos adquiridos desde los saberes del territorio, además se expresan en la preparación y disposición de los muebles para evitar la obstrucción o dificultades de movilidad durante la evacuación, así como para resguardar los alimentos de caídas durante el terremoto. En consecuencia, se logra mayor robustez mediante la implementación de ajustes al interior de los hogares de las familias. En los relatos se observa que en aquellas viviendas no equipadas para evitar la caída de muebles y el quiebre de vidrios, miembros de las familias sufren cortes en la piel y en los pies, lo que genera complicaciones para la evacuación y en las posteriores acciones para el abastecimiento.

Durante las acciones familiares para evacuar luego del terremoto, se evidencia el rol doméstico de la esposa (Tobin-Gurley y Enarson, 2013; en Thomas, 2013, p. 149; Enarson et al. 2014; en Rodríguez et al., 2014: p. 142) en el logro de una respuesta rápida. El conocimiento y manejo de la ubicación y disposición de los recursos al interior de la vivienda, le permite acceder con rapidez (Norris et al., 2007) a los insumos necesarios para la fase siguiente de la evacuación, tales como la ropa, botellas y bolsos. La distinción de género respecto a la responsabilidad del funcionamiento domestico genera dependencia por parte del hombre, haciendo más lenta su reacción. 
En la evacuación al tsunami, el compromiso de los vecinos por el bienestar de los miembros de la comunidad facilita que las acciones colectivas se orienten a compartir los recursos propios para ayudar a reducir la vulnerabilidad de otros (Kulig, 2008, en Ainuddin and Routray, 2012). Es así como una entrevistada de la unidad vecinal El Manzano, evacúa junto a su familia en el microbús de un vecino, quien en el trayecto hacia una zona segura, invita a subir a otros vecinos que evacuaban a pie.

De igual modo, el sentido de comunidad de los vecinos facilita que estos adquieran un rol fundamental en la actualización y entrega de información respecto a la búsqueda de familiares, ayudando a reducir la ansiedad y lograr el reencuentro entre los miembros de las familias.

Luego del terremoto y de la evacuación al tsunami, se inicia una crisis social vinculada a una percepción pesimista del desastre y a la sostenibilidad de la vida comunitaria, las que se expresan en dos situaciones: la emergencia de una multitud que ingresa a supermercados para extraer los productos de las estanterías, y el rumor sobre el tránsito de personas armadas, denominadas hordas, provenientes de otros lugares con intención de robar las viviendas.

"[ ] porque yo vivo en la calle como central, entonces ahí, mis vecinos pasaban, pasaban, incluso hasta con máquinas de ejercicio y todo, y mucha gente lo hacía, con camioneta todo, [...] los vecinos estaban totalmente (2) como te puedo explicar a ver (1) eran otros, después vino toda esa masa de-, de que iban a entrar a, a saquear las caaaasas y que había que ordenarse, el vandalismo (1) que hubo (1) hacia todo el sector de Talcahuano pu."' [código de caso: AC:20161017]

Los saqueos a supermercados efectuados por la multitud, se vinculan a una racionalidad moral que corre el riesgo de quedar entrampada en un reduccionismo económico que desestime la complejidad de las motivaciones y las condiciones previas al estímulo de la escasez. En vista a lo cual, la moral de la economía de subsistencia planteada por Thomson (1995) nos facilita la comprensión de los supuestos morales respecto al rol público del mercado durante situaciones de crisis y la validación del saqueo a partir de la clasificación positiva del acto de compartir los recursos sustraídos.

La multitud que emerge durante el desastre, actúa bajo la validación de una estructura moral de subsistencia que considera el saqueo un medio aceptado para dar solución a la escases luego del impacto del tsunami y producto de la baja preparación familiar para lograr una mayor autonomía. Incluso la transferencia de la información sobre los puntos de abastecimiento y saqueo adquieren un carácter solidario entre las familias. No obstante, el saqueo pierde calidad moral cuando no se genera la distribución o reparto de los recursos, cuando no existe regulación sobre la prioridad de los insumos sustraídos y cuando se produce acaparamiento de quien saqueó (Thomson, 1995).

Por su parte, la aceptación del rumor y la proliferación de historias ad-hoc respecto al desplazamiento de grupos de personas, denominadas hordas, con el objetivo común de robar las viviendas, obedece a la ausencia de una fuente oficial de información que comunique los procesos de respuesta de los organismos de emergencia (Scanlon, 2014; en Rodríguez, 2014, p. 426) y las coordinaciones respecto a la ayuda humanitaria. En consecuencia, el desconocimiento respecto a las capacidades de respuesta de los organismos públicos y de emergencia, junto con la desconfianza generada hacia ellos por la entrega de información errónea sobre la alerta de tsunami, facilita el aumento en la sensación de desprotección, especialmente durante las primeras noches sin luminarias públicas producto a la caída del tendido eléctrico.

En respuesta al contexto del rumor, las familias comparten la necesidad de proteger sus hogares mediante atrincheramientos con fogatas y turnos de relevo en guardias nocturnas ubicadas en los accesos de los pasajes. Esta acción colectiva está caracterizada por operaciones de carácter bélicas (Dynes, 1994), tales como la presencia de armas de fuego, el control de los ingresos y salidas, y por la definición de etiquetamientos para la identificación de los vecinos. No obstante, en torno a las fogatas y las guardias nocturnas se produce una mayor participación comunitaria, la cual es recordada con nostalgia por los entrevistados. 
Por consiguiente, durante las acciones vinculadas al campo de acción de la seguridad, aumenta el sentido de pertenencia, lo cual ayuda a disminuir la sensación de vulnerabilidad y desprotección. Sin embargo, también se genera indiferencia y hostilidad hacia miembros de otras comunidades cercanas (Aldrich, 2012), quienes responden a clasificaciones previas respecto a la peligrosidad de poblaciones cercanas, las que son vistas como una amenaza.

En vista al desempeño de las capacidades comunitarias de afrontamiento, las vulnerabilidades relacionadas al estado de salud (Cutter, 2003; Adger et al., 2004; Bergstrand et al., 2014) y la edad (Laska et al. 2005; Lee et al., 2014; Singh, 2014), sobresalen en cuanto obstaculizadores para una respuesta rápida y autónoma. En los relatos destacan las dificultades para despertar durante el terremoto y reaccionar para una rápida la evacuación, en quienes habían consumido medicamentos para conciliar el sueño. En cuanto a la edad, las primeras preocupaciones de los entrevistados luego del terremoto, son los bebes y los adultos mayores quienes reciben y requieren mayor asistencia para evacuar de las viviendas.

Se sintetiza lo anteriormente presentado en la Figura 2, que es encabezada por una línea temporal del desastre, considerando dos momentos temporales: las primeras 72 horas inmediatamente posteriores al terremoto y tsunami (período de respuesta), y el período de entre 1 y 3 meses posteriores al desastre (período de recuperación). Se relacionan estas etapas con las capacidades adaptativas y de afrontamiento que demostró la comunidad.

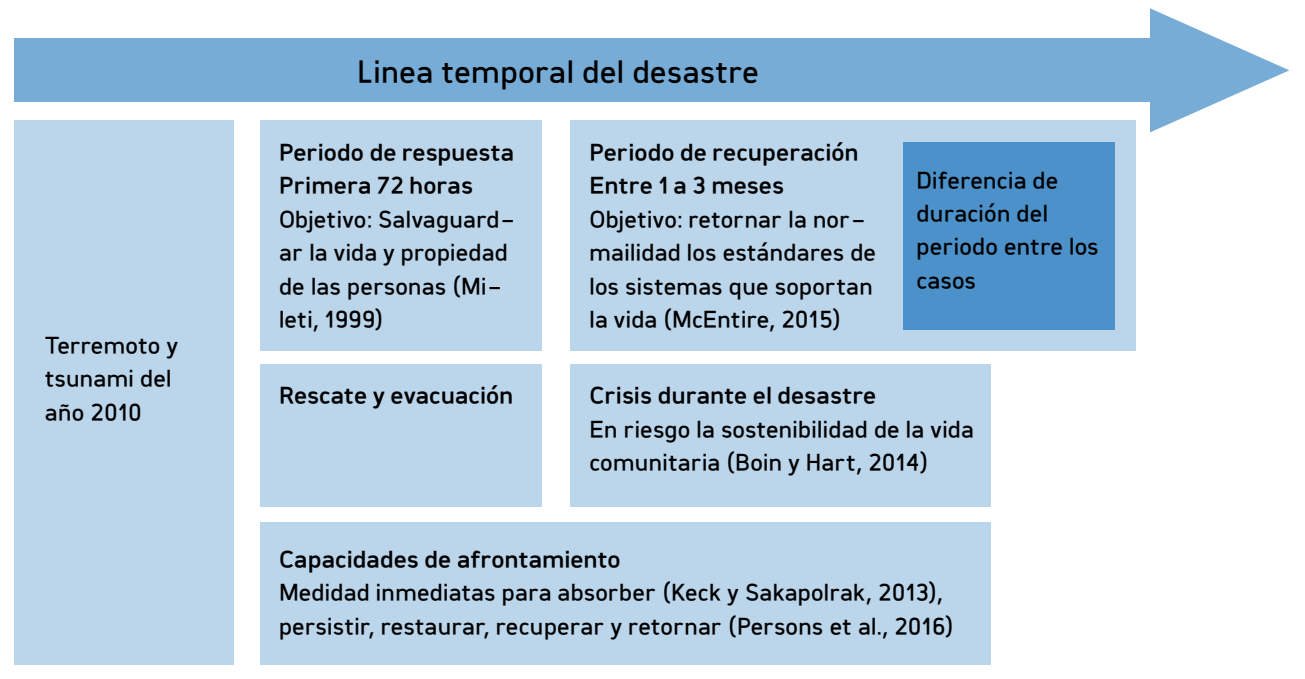

Figura 2: Línea temporal de los periodos de respuesta y recuperación, vinculados con las capacidades de afrontamiento y las capacidades adaptativas.

Fuente: Autores, 2018

\section{Capacidades de afrontamiento durante el periodo de recuperación}

El periodo de recuperación se define como el momento en que las acciones están enfocadas en retornar a la normalidad los estándares de los servicios que soportan la vida (Mileti, 1999). De modo que luego de las primeras 72 horas del desastre, con el arribo de la fuerza militar en el territorio y el consiguiente toque de queda, las acciones comunitarias e institucionales se enfocan principalmente en las tareas de distribución de alimento, agua y las acciones de limpieza de calles.

La duración del periodo depende del grado de exposición al tsunami y de la accesibilidad hacia los sectores inundados que determina la visibilidad de la afectación. En sectores más próximos a la costa, la normalidad del suministro de agua potable tomó casi tres veces más tiempo que en sectores más cercanos a la vialidad estructural de la comuna.

No obstante, luego de la evacuación se inicia una fase de crisis, instancia en la que se presentan diferentes estadios de vinculaciones, los que podrían observarse como diferentes campos de acción (Bourdieu, 1979) de los atributos dinámicos de los recursos (Norris et al., 2007) comunitarios 
que permiten la resiliencia comunitaria, mientras sucedían los saqueos desde la multitud, algunos entrevistados estaban realizando gestiones para abastecerse de agua, otros se organizaban con sus vecinos para resguardar sus viviendas.

Entre los casos inundados que perdieron las condiciones de habitabilidad de sus viviendas, las familias y amistades son el principal soporte para la emergencia habitacional. Por lo que, las familias migran de sus viviendas y no regresan hasta terminar con las acciones de limpieza y desinfección del fango; y la eliminación de muebles, electrodomésticos hacia a las calles. Esta capacidad para afrontar la crisis es principalmente familiar y no comunitaria, mientras que la gestión de vivienda de emergencia para quienes se les destruyó el inmueble, fue una gestión comunitaria y política.

Se desprende de la discusión de los resultados, que en las familias que expresan mayor preocupación por el bienestar de los otros vecinos, sus miembros tienden a participar en acciones altruistas y adquirir roles de liderazgo en acciones colectivas con el objeto de resolver los problemas de la manera más eficiente.

"[ ] estaban construyendo unos edificios [ ] Entonces estaban drenando agua. Y ahí habían unas motobombas compadre, y salían así un chorro de agua, entonces un día me pasaron el dato y yo fui pa' allá po'. Y empiezo a cachar que el ducto de agua que entregaba era así, y las botellitas eran así po. Ya entonces fui pa' delante y le dije al socio, 'socio, estamos mal' y mejoramos el sistema [ ] hicimos embudos, pa' ir llenando más rápido [ ] empezamos a agilizar [ ]. Pero después de toda la pega si po'. Agilizamos la entrega. Oye, después tú te podías ir a bañar ahi”. [código de caso: JM:20161018]

De igual modo, el sentido de comunidad está ligada a los años en el territorio y a las experiencias compartidas, lo cual facilita la gestación de organización comunitaria producto de la confianza (Aldrich, 2012), el reconocimiento de historias comunes, incluso si por años no hubiese existido relación. El aumento de la participación de las familias en las acciones colectivas permite un mayor desempeño de las capacidades de afrontamiento al facilitar el acceso a recursos comunitarios y familiares. No obstante, las personas adultos mayores tienden a quedar excluidas de la emergencia de las redes de apoyo que surgen durante la crisis de abastecimiento.

En aquellos casos donde el sentido de comunidad es menor producto del autoaislamiento y la desconfianza social, las acciones colectivas fueron menos frecuentes e incluso cargadas de malos entendidos o conflictos que se desprenden de las expectativas de la población respecto de la asistencia pública y del rol de los dirigentes comunitarios.

El rol de la dirigencia en las juntas de vecinos tiende a quedar en manos de mujeres, quienes además de gestionar la ayuda humanitaria para la comunidad y adquirir un rol de liderazgo sobre la resolución de los problemas al interior de las comunidades, continúan con las funciones de soporte familiar durante tiempos normales. Mientras que en las poblaciones sin juntas de vecinos, la acción comunitaria presenta mayores dificultades y requieren del apoyo de aquellas comunidades que cuentan con una organización comunitaria establecida.

La acción colectiva surge entre los vecinos sin que haya existido anteriormente vinculación o trabajo conjunto. No obstante, los ajustes y mejoras de las soluciones colectivas son facilitados por la actuación de los liderazgos locales preexistentes, quienes identifican y resuelven los problemas enfocados en el mayor desempeño de los recursos comunitarios.

Aunque aumenta el sentido de comunidad durante la crisis, luego de la normalización de los servicios, la frecuencia de las relaciones decae y se retorna a la normalidad donde la participación entre los vecinos es escasa y la desconfianza entre ellos es una condición generalizada entre los casos de estudio. No obstante, luego del periodo de recuperación se conforman nuevas juntas de vecinos en sectores que no contaban formalmente con organización comunitaria.

Se observa que al interior de las comunidades se generan acciones de cooperación para implementar soluciones improvisadas a problemas para los cuales no estaban preparados 
colectivamente, quebrando el modelo tradicionalista que considera a las personas como entidades frágiles que requieren de respuestas centralizadas y controladas. Por el contrario, las acciones colectivas cuyos estilos de resolución de problemas están caracterizados por la delegación de responsabilidades entre los integrantes de la comunidad, permite reducir el desgaste de los dirigentes comunitarios y acelerar la distribución de los recursos.

La recuperación de las familias logra un mayor desempeño mediante acciones de cooperación y de ayuda entre vecinos, mientras que aquellas comunidades cuyas familias presentan menos participación en las organizaciones emergentes, requieren de más tiempo y de un mayor desgaste físico y emocional para retornar a la normalidad. Especialmente las familias inundadas por el tsunami, las que tienen que limpiar ellas mismas sus viviendas, esporádicamente ayudados por voluntarios de organizaciones externas al territorio.

Esposo: "yo me acuerdo que ese día del terremoto yo compré algunas cosas, me aseguré con algunas cosas más [ ] En un supermercado que hay allí [ ] no fueron tan amarretes, no se aprovechaban. Así que me aseguré con varias cosas ahí. Para tener.[ ] Longanizas [risas]. ¿Por qué? Porque esas cuestiones se echan a perder." [Código de caso: RP:20161018]

Incluso, la capacidad comunitaria para afrontar la escases de alimentos logra una mayor independencia y redundancia en el uso recursos, cuando los propios miembros adquieren un compromiso por amortiguar la afectación en los miembros de la comunidad. Prueba de ello fue el rol comunitario de algunos almacenes de abarrotes que continuaron sus operaciones durante los primeros días del desastre, lo que permite que familias cercanas se abastezcan de alimentos, en la mayoría de los casos, al mismo precio que antes del desastre. Por consiguiente, cuando el sentido de comunidad es menor producto del poco interés por el bienestar de la comunidad, se generan prácticas abusivas por parte de algunos vecinos que buscan beneficiarse de la escases y del mercado negro.

Mediante la investigación se logra dar cuenta que no bastan las capacidades familiares para responder durante la primeras fases del desastre, ocurrencia del terremoto y evacuación. Se requiere desarrollar capacidades comunitarias para afrontar colectiva y eficientemente el quiebre de los servicios mediante el uso de los recursos comunitarios disponibles en el territorio. En virtud de lo cual el sentido de comunidad, expresado por el compromiso por el bienestar de la comunidad y la habilidad para resolver problemas colectivamente, aumenta el desempeño al acceso a redes de apoyo y a la ayuda humanitaria.

Para finalizar, la Figura 3 organiza las capacidades de afrontamiento identificadas para cada uno de los períodos considerados en esta investigación (período de respuesta y período de recuperación). Las capacidades de afrontamiento corresponden a los rectángulos grises en la mitad inferior del cuadro. Agrupando a estas capacidades se pueden encontrar varios cuadros verdes, que representan distintos componentes del sentido de comunidad, que involucran y movilizan a estas diferentes capacidades de afrontamiento. El cuadro celeste, en cambio, representa a la memoria social, que se considera una capacidad adaptativa en tanto espacio que facilita el aprendizaje y la transmisión de conocimiento.

\section{CONCLUSIONES}

La investigación plantea la pregunta respecto a cómo se expresan las capacidades de afrontamiento vinculadas a la resiliencia comunitaria ante desastre, en relación al sentido de comunidad en familias residentes en el territorio de Las Salinas en la comuna de Talcahuano durante el periodo de respuesta y recuperación al desastre producto del terremoto y tsunami del 27 de febrero del año 2010

En vista a la discusión teórica se argumenta que la resiliencia comunitaria se constituye por los atributos de las capacidades de un sistema para afrontar y adaptarse a situaciones de desastre con el objeto de resistir el impacto de un desastre y generar los ajustes para mejorar las condiciones de adaptabilidad de la comunidad ante futuras situaciones de crisis. No obstante, la resiliencia comunitaria requiere de ciertas condiciones sociales vinculadas con el sentido de comunidad para lograr un mayor desempeño en el uso de los recursos locales durante el desastre. 


\section{CAPACIDADES DE AFRONTAMIENTO}

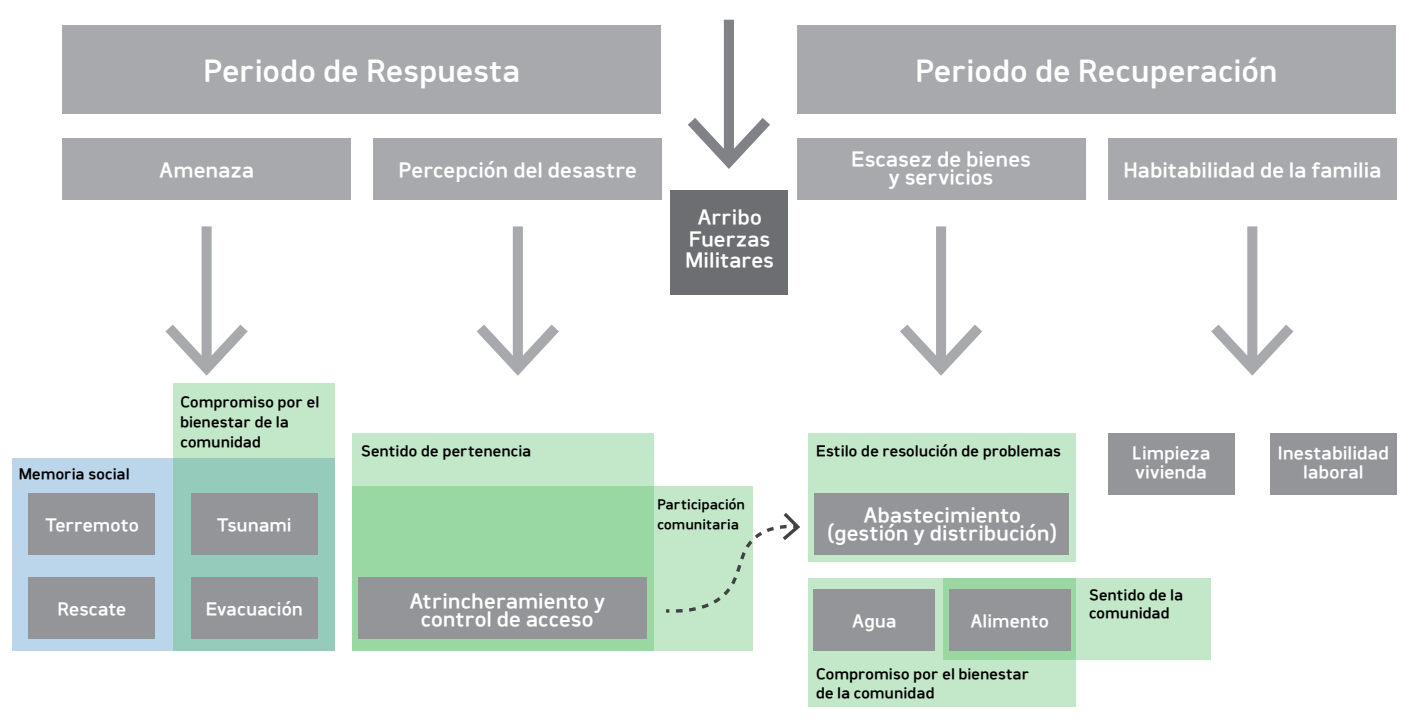

Figura 3: Capacidades de afrontamiento vinculados con el sentido de comunidad durante el periodo de respuesta y recuperación Fuente: Autores, 2018

El análisis de datos realizado a los discursos de trece casos de estudio da cuenta que la resiliencia comunitaria es un proceso que emerge de la vinculación con el sentido de comunidad de los residentes de un territorio, lo cual se expresa en el compromiso por el bienestar de la comunidad del lugar (Kulig et al. 2008 citado en Ainuddin y Routay 2012, p. 28), el sentido de pertenencia y arraigo al territorio (Roterson, 2000 y Allen, 2003, citado en Wilson, 2012, p. 83) y en la participación comunitaria (Pfferfferbaun et al., 2015) en la resolución de problemas colectivos (Kenney et al. 2015 , p. 48). Estas condiciones facilitan la organización social y el surgimiento de comportamientos colaborativos (Norris et al., 2007) en función de resolver los problemas con mayor rapidez y desempeño en el uso de los recursos del territorio (Longstaff et al. 2010).

Las capacidades de afrontamiento de la resiliencia comunitaria forman parte de un mismo proceso de mejoramiento y adaptación de las condiciones sociales que obstruyen la respuesta cooperativa y la reducción de las vulnerabilidades ante desastre. En virtud de lo cual, la memoria social transmitida oralmente al interior de las familias, en algunos casos traducidas en prácticas de almacenamiento y preparación familiar, fue determinante en la capacidad para afrontar los primeros días de la crisis del desastre. En consecuencia, los aprendizajes sociales obtenidos con la experiencia del desastre estudiado, quedan sensiblemente contenidos en la memoria de las familias. Así como lo hicieron anteriormente las personas mayores, esta generación son los nuevos contenedores de un conjunto de conocimientos que requieren ser rescatados a través de acciones colaborativas y participativas.

La investigación pone en evidencia que no bastan las capacidades familiares para responder durante las primeras fases del desastre, (ocurrencia?)y evacuación, ni durante la crisis de escases. Se requiere desarrollar capacidades comunitarias para afrontar colectiva y eficientemente el quiebre de los servicios mediante el uso de los recursos comunitarios disponibles en el territorio (Magis, 2010, p. 410-411). De esta forma, aumentar el sentido de comunidad ante desastres, para facilitar el acceso a nuevas redes de apoyo, a la ayuda humanitaria y a los recursos comunitarios del territorio.

Especialmente, cuando la responsabilidad del bienestar de la comunidad y del desempeño de los procesos de gestión y distribución de la ayuda humanitaria, recae principalmente sobre los 
dirigentes vecinales de las juntas de vecinos, principalmente mujeres, que no contaban con entrenamiento previo, ni con los recursos para llevar a cabo las tareas de diagnóstico, comunicación, contención emocional o gestión política de los recursos. Aun así, el robusto compromiso de los líderes comunitarios por el bienestar de los residentes del territorio, motiva que los dirigentes de una Junta de Vecinos adicionen a su gestión la responsabilidad por el bienestar de vecinos de otras poblaciones del territorio sin organización comunitaria de carácter territorial.

La capacidad de la comunidad para adaptarse organizativamente en torno a la participación en acciones colectivas vinculadas al contexto de la seguridad durante la crisis, responde a un aumento del sentido de pertenencia al territorio y del compromiso por el bienestar de la comunidad del lugar. Aunque, cuando se resuelve la crisis del abastecimiento de agua, electricidad y alimento, las familias retornan a una estado de escasa participación. Sin embargo, así como aumenta el sentido de comunidad, también se genera indiferencia y hostilidad a miembros de otras comunidades cercanas (Aldrich, 2012), las que responden a clasificaciones previas respecto a la peligrosidad de poblaciones fuera del territorio.

El aumento de la sensación de inseguridad y desprotección producto de los rumores, facilita el surgimiento de una acción colectiva caracterizada por operaciones de carácter bélicas, tales como atrincheramientos con fogatas, la presencia de armas de fuego y la definición de etiquetas para la identificación de los vecinos. Lo cual deriva de una percepción del desastre como un homólogo de un estado de guerra, analogía que deriva de la participación en organismos de las fuerzas armadas o militares (Dynes, 1994).

Los aprendizajes sociales adquiridos con el desastre, requieren de iniciativas que desarrollen estratégicamente las habilidades para mejorar la participación de las familias residentes en acciones colectivas (Tierney, 2014, p. 182; Magis, 2010) y del incremento del sentido de arraigo con la comunidad del territorio (Berke y Campanella, 2006; Roterson, 2000 y Allen, 2003, en Wilson, 2012, p. 83) para que los ajustes sean creaciones desde las mismas familias del lugar (Mileti, 1999). De la calidad de estos aprendizajes depende el fortalecimiento de las capacidades de afrontamiento para anticiparse a futuras situaciones de desastre. En este aspecto, los liderazgos preexistentes (Kenney 2015, p. 48) logran favorecer el mejor desempeño del uso de los recursos locales (Tierney, 2003, p. 40), mediante estilos de resolución de problemas centrados en la delegación de responsabilidades y en la promoción de la participación comunitaria (Pfefferbaun et al. 2015).

En cuanto a las vulnerabilidad sociales que perjudican la capacidades para afrontar el desastre, se concluye que el aislamiento de las personas de mayor edad, la dependencia y uso de medicamentos, la desigualdad género en los roles domésticos, la escasa robustez interna de la vivienda, el desconocimiento de las condiciones de riesgo del territorio, y la escasa participación comunitaria de los vecinos en acciones colaborativas, limitan una respuesta familiar rápida, robusta y redundante.

En consecuencia, los habitantes necesitan prepararse cooperativamente y anticiparse a los riesgos con el objeto de operar de manera más autónoma que como lo eran antes del desastre (Drabek, 2014 en Rodríguez, 2014, p. 220). A través de la promoción del sentido de comunidad ante desastre, es posible facilitar la resiliencia comunitaria y reducir la susceptibilidad ante desastres, cuyas amenazas no siempre son controlables.

\section{BIBLIOGRAFIA}

Adger, N. (2006). Vulnerability. Global Environmental Change, 16, 268-281.

Ainuddin \& Routray (2012). Community resilience framework for an earthquake prone area in Baluchistan. International Journal of Disaster Risk Reduction, 2, 25-36.

Aldrich (2012) Building Resilience: Social Capital in Post Disaster Recovery. Chicago: University of Chicago Press.

Altman \& Low (2012) Place Attachment. New York: Springer.

Angermann, D., Klotz, J. \& Reigber C. (1999). Space-geodetic estimation of the Nazca-South America 
Euler vector. Earth and Planetary Science Letters, 117, pp.329-334.

Bankoff, G., Frerks, G., \& Hilhorst, T. (2006). Mapping vulnerability: disasters, development, and people. London: Earthscan Publications.

Bergstrand, K., Mayer, B., Brumback, B. \& Zhang, Y. (2014). Assessing the Relationship Between Social Vulnerability and Community Resilience to Hazards. Social Indicators Research, 122, pp.391-409.

Berke \& Campanella (2006). Planning for post-disaster resiliency. Annals of the American Academy of Political and Social Science, 604, pp.192-207.

Berkes \& Ross (2013). Community Resilience: Toward an Integrated Approach. Society E Natural Resources, 26, pp.5-20.

Blaikie, P., Cannon, T., Davis, I. \& Wisner, B. (1994). At Risk: Natural Hazards, People’s Vulnerability, and Disasters. London: Routledge.

Bruneau, M., Chang, S., Eguchi, R., Lee, G., O’Rourke, T., Reinhorn, A., Shinozuka, M., Tierney, K., Wallace, W. \& Von Winterfeldt, D. (2003). A Framework to Quantitatively Assess and Enhance the Seismic Resilience of Communities. Earthquake Spectra, 19(4), pp.733-752.

Cannon, T. (1994). Vulnerability Analysis and the Explanation of "Natural” Disasters. (A. Varley, Ed.) Disasters, Development and Environment. Chichester, UK: John Wiley and Sons Lld.

Chamlee-wright \& Storr (2009). 'There's No Place Like New Orleans': Sense of Place and Community Recovery in the Ninth Ward after Hurricane Katrina. Journal of Urban Affairs, 31(5), pp. 615-634.

Chang (2010). Community cohesion after a natural disaster: Insights from a Carlisle flood. Disasters, 34(2), pp.289-302.

Cisternas, M. (2012). El terremoto de 1647 de Chile central como un evento intraplaca : ¿otra amenaza para Chile metropolitano?. Revista de Geografía Norte Grande, 53, pp.23-33.

Cisternas, M., Atwater, B., Torrejón, F., Sawai, Y., Machuca, G., Lagos, M., Eipert, A., Youlton, C., Salgado, I., Kamataki, T., Shishikura, M., Rajendran, C., Malik, J., Rizal, Y. \& Husni, M. (2005). Predecessors of the giant 1960 Chile earthquake. Nature, 437, pp.404-407.

Cohen, O., Goldberg, A., Lahad, M. \& Aharonson-Daniel, L. (2016). Building resilience: The relationship between information provided by municipal authorities during emergency situations and community resilience. Technological Forecasting and Social Change, 121, pp.119-125.

Cutter, S., Emrich, C., Webb, J. \& Morath, D. (2009). Social Vulnerability to Climate Variability Hazards: A Review of the Literature. Final Report to Oxfam America. Hazard and Vulnerability Research Institute. Columbia: University of South Carolina.

Cutter, S., Barnes, L., Berry, M., Burton, C., Evans, E. \& Tate, E. (2008). A place-based model for understanding community resilience to natural disasters. Global Environmental Change, 18(4), pp.598-6o6.

Cutter, S. Boruff, B. \& Shirley, W. (2003). Social vulnerability to environmental hazards. Social Science Quarterly, 84(2), pp.242-261.

Dombrowsky, W. (1995). Again and Again: Is a Disaster What We Call “Disaster”? Some Conceptual Notes on Conceptualizing the Object of Disaster Sociology. International Journal of Mass Emergencies and Disasters, 13(3), pp.241-254.

Drabek, T. (1970). Methodology of Studying Disaster. American Behavioral Scientist, 13(3), pp.331-343.

Dynes, R.(2005). Community social capital as the primary basis of resilience, University of Delawer Disaster Research Center, Preliminary Paper \#344.

Dynes, R. (1994). Community Emergency Planning: False Assumption and Inappropriate Analogies. International Journal of Mass Emergencies and Disasters, 12(2), pp.141-158.

Emrich, C. \& Cutter, S. (2011). Social Vulnerability to Climate-Sensitive Hazards in the Southern United States. American Meteorological Society, 3, pp.193-208.

Fakhruddin, S. \& Rahman, J. (2015). Coping with coastal risk and vulnerabilities in Bangladesh. International Journal of Disaster Risk Reduction, 12, pp.112-118.

Fraser, M., Galinsky, M. \& Richman, J. (1999). Risk, protection and resilience: Toward a conceptual framework for social work practice. Social Work Research, 23(3), pp.131-143.

Henríquez, G. \& Barriga, O. (2005). El Rombo de la Investigación. Cinta Moebio, 23, pp.162-168.

Greimas, J. (1966). Semántica Estructural: Investigación Metodológica (3era Ed.). Madrid: Gredos. 
Hiernaux, J. (1996). Análisis estructural de contenidos y de modelos culturales. Lovaina: Universidad de Lovaina.

Holling, C. (1994). Simplifying the complex: The paradigms of ecological function and structure. Futures, 30, pp.139-46.

Keck, M. \& Sakdapolrak, P. (2013). What is social resilience? lessons learned and ways forward. Erdkunde, 67, pp.5-19.

Kenney, C. \& Phibbs, S. (2015). A Māori love story: Community-led disaster management in response to the Ōtautahi (Christchurch) earthquakes as a framework for action. International Journal of Disaster Risk Reduction, 14, pp.46-55.

Krippendorf, K. (2004). Content analysis: an introduction to its methodology (2da Ed.). Thousand Oaks: Sage Publications.

Laska, S. \& Hearn Morrow, B. (2005). Social Vulnerability and Hurricane Katrina: An Unnatural Disaster in New Orleans. Marine Technology Society Journal, 40(4), pp. 16-26.

Lee, Y. (2014). Social vulnerability indicators as a sustainable planning tool. Environmental Impact Assessment Review, 44, pp.31-42.

Longstaff, P., Armstrong, N., Perrin, K., Parker, W. \& Hidek, M. (2010). Building Resilient Communities: A Preliminary Framework for Assessment. Homeland Security Affairs, 4(3), pp.1-23.

Magis, K. (2010). Community Resilience: An Indicator of Social Sustainability. Society E Natural Resources: An International Journal, 23, pp.401-416.

Manyena, B., O’Brien, G., O’Keefe, P. \& Rose, J. (2011). Disaster resilience: a bounce back or bounce forward ability? Local Environment, 16(5), pp.417-424.

McEntire, D. (2014). Disaster Response and Recovery. Strategies and Tactics for Resilience (2da Ed.). New Jersey: Wiley.

McMillan, D. \& Chavis, D. (1986). Sense of community: A definition and theory. Journal of Community Psychology, 14, pp.6-23.

Melnick, D., Cisternas, M., Moreno, M. \& Norambuena, R. (2012). Estimating coseismic coastal uplift with an intertidal mussel: Calibration for the 2010 Maule Chile earthquake $(\mathrm{M} \mathrm{w}=8.8)$. Quaternary Science Reviews, 42, pp. 29-42.

Mileti, D. (1999). Disasters by design: A reassessment of natural hazards in the United States. Washington, DC: Joseph Henry Press.

Moreno, M., Melnick, D., Rosenau, M., Baez, J., Klotz, J. \& Oncken, O. (2012). Toward understanding tectonic control on the M w 8.82010 Maule Chile earthquake. Earth and Planetary Science Letters, 321, pp.152-165.

Municipalidad de Talcahuano. (2017). Sistema de Información Geográfica para la Gestión del Riesgo Revisado el día domingo 21 de mayo de 2017 http://riesgo.talcahuano.cl/.

Narváez, L. Lavell, A. \& Pérez, G. (2009). La Gestión del Riesgo de Desastres: Un enfoque basado en procesos. Lima: PREDECAN.

Norris, F., Stevens, S., Pfefferbaum, B., Wyche, K., \& Pfefferbaum, R. (2007) Community Resilience as a Metaphor, Theory Set of Capacities and Strategy for Disaster Readiness. American Journal of Community Psychology, 41, pp.127-150.

Parsons, M., Glavac, S., Hastings, P., Marshall, G., Mcgregor, J., Mcneill, J., Morley, P., Reeve, I. \& Stayner, R. (2016). Top-down assessment of disaster resilience: A conceptual framework using coping and adaptive capacities. International Journal of Disaster Risk Reduction, 19, pp.1-11.

Paton, D., Millar, M. \& Johnston, D. (2001). Community resilience to volcanic hazard consequences. Natural Hazards, 24, pp.157-169.

Patterson, O., Weil, F. \& Patel, K. (2009). The role of community in disaster response: Conceptual models. Population Research and Policy Review, 29, pp.127-141.

Perkins, D., Hughey, J. \& Speer, P. (2002). Community psychology perspectives on social capital theory and community development practice. Community Development, 33, 33-52.

Pfefferbaum, B., Pfefferbaum, R. \& Van Horn, R. (2015). Community Resilience Interventions: Participatory, Assessment-Based, Action-Oriented Processes. American Behavioral Scientist, 59(2), pp.238-253. 
Quesada (2004). El enfoque de resiliencia en Trabajo Social. Departamento de Trabajo Social Y Ciencias Sociales. Sevilla: Universidad Pablo de Olvide.

Rodríguez, H., Quarantelli, E. \& Dynes , R. (2014). Handbook of Disaster Research. Texas: Springer.

Sapirstein, G. (2006). Social Resilience : The Forgotten Dimension of Disaster Risk Reduction. Jamba: Journal of Disaster Risk Studies, 1, pp.54-63.

Singh, S., Eghdami, M. \& Singh, S. (2014). The Concept of Social Vulnerability : A Review from Disasters Perspectives. International Journal of Interdisciplinary and Multidisciplinary Studies, 1(6), pp.71-82.

Smit, B. \& Wandel, J. (2006). Adaptation, adaptive capacity and vulnerability. Global Environmental Change, 16, pp.282-292.

Stallings, R. (2002). Methods of Disaster Research. International Research Committee on Disaster. Philadelphia: Xlibris.

Thomas, D., Phillips, B., Fothergill, A. \& Blinn-Pike, L. (2013). Social vulnerability to disasters. Boca Raton, FL: CRC Press.

Thompson, E. (1995). Costumbres en común. Barcelona: Critica.

Tierney, K. (2003). Disaster Beliefs and Institutional Interests: Recycling Disaster Myths in the Aftermath of 9-11. Research in Social Problems and Public Policy, 11, pp.33-51.

Tierney, K. (2014). The social roots of risk. Producing disaster, promoting resilience. California: Stranford Business Books \& Stanford University Press.

Turner, B., Kasperson, R., Matson, P., McCarthy, J., Corell, R., Christensen, L., Eckley, N., Kasperson, J., Luers, A., Martello, M., Polsky, C., Pulsipher, A. \& Schiller, A. (2003). A framework for vulnerability analysis in sustainability science. Proceedings of the National Academy of Sciences of the United States of America, 100(14), pp. 8074-8079.

UNISDR. (2015). Marco de Sendai para la Reducción del Riesgo de Desastre 2015-203o. Tercera Conferencia Mundial de las Naciones Unidas sobre la Reducción del Riesgo de Desastres. Sendai: UNISDR.

UNISDR. (2005). Marco de acción de Hyogo para 2005-2015. Kobe, Hyogo: UNISDR.

Vieytes, R. (2004). Metodología de la investigación en organizaciones, mercado y sociedad. Buenos Aires: De las ciencias.

Wamsler, C. (2014). Cities, disaster risk and adaptation. London \& New York: Routledge Taylor and Francis Group.

Werner, E. \& Smith, R. (1982). Vulnerable But Invincible: A Study of Resilient Children and Youth. New York: McGraw-Hill.

Wesson, R., Melnick, D., Cisternas, M., Moreno, M. \& Ely, L. (2015). Vertical deformation through a complete seismic cycle at Isla Santa María, Chile. Nature Geoscience, 8, pp.547-551.

Wilson, G. (2012). Community Resilience and Environmental Transition. New York: Routledge.

Wisner, B., Blaikie, P., Cannon, T., \& Davis, I. (2004). At risk : natural hazards, people's vulnerability, and disasters (2nd ed.). London \& New York: Routledge. 